\title{
A retrospective analysis of ultrasound-guided large core needle biopsies of breast lesions at a regional public hospital in Durban, KwaZulu-Natal, South Africa
}

\begin{tabular}{|c|c|}
\hline \multicolumn{2}{|c|}{$\begin{array}{l}\text { Authors: } \\
\text { Schene Bhayroo }{ }^{1} \\
\text { Leon Yaseen Perumal }^{1} \\
\text { Ines Buccimazza }\end{array}$} \\
\hline \multicolumn{2}{|c|}{$\begin{array}{l}\text { Affiliations: } \\
{ }^{1} \text { Department of Radiology, } \\
\text { University of KwaZulu-Natal, } \\
\text { South Africa }\end{array}$} \\
\hline \multicolumn{2}{|c|}{$\begin{array}{l}{ }^{2} \text { Department of Surgery, } \\
\text { University of KwaZulu-Natal, } \\
\text { South Africa }\end{array}$} \\
\hline \multicolumn{2}{|c|}{$\begin{array}{l}\text { Corresponding author: } \\
\text { Schene Bhayroo, } \\
\text { s.bhayroo@gmail.com }\end{array}$} \\
\hline \multicolumn{2}{|c|}{$\begin{array}{l}\text { Dates: } \\
\text { Received: } 26 \text { Feb. } 2015 \\
\text { Accepted: } 07 \text { June } 2016 \\
\text { Published: } 27 \text { July } 2016\end{array}$} \\
\hline \multicolumn{2}{|c|}{$\begin{array}{l}\text { How to cite this article: } \\
\text { Bhayroo S, Perumal, LY. A } \\
\text { retrospective analysis of } \\
\text { ultrasound-guided large core } \\
\text { needle biopsies of breast } \\
\text { lesions at a regional public } \\
\text { hospital in Durban, } \\
\text { KwaZulu-Natal, South Africa. } \\
\text { S Afr J Rad. 2016;20(1), a994. } \\
\text { http://dx.doi.org/10.4102/ } \\
\text { sajr.v20i1.994 }\end{array}$} \\
\hline \multicolumn{2}{|c|}{$\begin{array}{l}\text { Copyright: } \\
\text { (C) 2016. The Authors. } \\
\text { Licensee: AOSIS. This } \\
\text { is licensed under the } \\
\text { Creative Commons } \\
\text { Attribution License. }\end{array}$} \\
\hline \multicolumn{2}{|l|}{ Read online: } \\
\hline 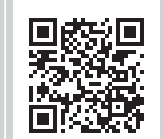 & $\begin{array}{l}\text { Scan this QR } \\
\text { code with your } \\
\text { smart phone or } \\
\text { mobile device } \\
\text { to read online. }\end{array}$ \\
\hline
\end{tabular}

Background: Histological confirmation of a breast lesion is an important step to determine the aetiology and direct further management. Evidence supports ultrasound-guided large core needle biopsy (US-LCNB) (14 gauge) as the preferred diagnostic method over traditional open surgical biopsy.

Objective: To assess the influence of technical variables on the diagnostic yield of breast specimens obtained by using US-LCNB, and the sensitivity of detecting malignancy during the study period.

Methods: A retrospective chart review was conducted of all patients who had US-LCNBs from March 2011 - September 2012 at Addington Hospital in Durban, KwaZulu-Natal. Histopathological findings were correlated to the size of the breast lesion, rank of the radiologist performing the procedure and the number of cores obtained. The sensitivity of the technique was determined.

Results: During the study period, 147 biopsies were performed. The majority of lesions were $>5 \mathrm{~mm}(85.5 \%)$. The average number of cores was 4 , and $79.5 \%$ of the biopsies were performed by the senior radiologist. Of the 147 biopsies, 132 specimens were eligible for inclusion in the study. Histopathology revealed 71 malignant lesions of which 60 were confirmed histologically at excision. In 11 patients, no excision was performed. Therefore, the sensitivity of detecting malignancy was $100 \%$.

Conclusion: Although the study did not establish a statistically significant relationship between the abovementioned technical variables and the histological outcome, the overall diagnostic yield and the sensitivity of detecting malignancy using US-LCNB is comparable to other similar international studies. A prospective study with long-term follow-up of patients would be of value.

\section{Introduction}

Mammography and ultrasonography aid in the characterisation of clinically palpable breast lesions and in screening for breast cancer. Histological confirmation of a breast lesion is an important step to determine the aetiology and direct further management. In South Africa, breast cancer constitutes a significant burden of disease, accounting for $20.82 \%$ of all cancer cases in the female population. ${ }^{1}$ Screening for breast cancer is best achieved by mammography. ${ }^{2,3}$ However, in South Africa there is no population-based mammographic screening programme. Where available, mammography is used to assess appropriately selected symptomatic patients. Ultrasonography is an important adjunct to mammography., ${ }^{4,5}$ It is a useful tool to characterise and biopsy symptomatic and occult breast lesions.

Evidence supports non-surgical percutaneous breast biopsy as the preferred diagnostic method over traditional open surgical biopsy. ${ }^{6,78}$ Non-surgical breast biopsies are less stressful, have a minimal risk of complications, do not cause architectural distortion of the skin or internal breast tissue that can interfere with the accuracy of future mammograms, and can be done as a day procedure. A pre-operative diagnosis of breast cancer provides the patient with all the treatment options available and permits an informed decision in respect of management. In the case of occult benign lesions, a non-surgical diagnosis reduces the incidence of unnecessary excision biopsies. ${ }^{8,9}$

The different types of non-surgical breast biopsy procedures include: fine needle aspiration biopsy (FNAB), core needle biopsy (CNB), vacuum-assisted breast biopsy (VABB) and stereotactic breast 
biopsy using mammographic guidance. FNAB is limited by targeting errors, bloody smears and the inability to subtype certain malignancies, resulting in false negative results. CNB may be performed either by clinical guidance (i.e. by palpation) or under image guidance using ultrasound, which has the advantage of real-time visualisation. VABB using ultrasound guidance has the advantage of obtaining multiple cores through a single skin insertion but cost is a prohibiting factor. Stereotactic breast biopsy exposes the patient to radiation, requires additional time to perform the procedure and skilled staff with adequate equipment - key factors which are commonly lacking in developing countries.

Ultrasound-guided large core needle biopsy (US-LCNB) using a 14-gauge biopsy needle benefits the resource-constrained setting in the developing world; however, the limitations of its use should not be overlooked. Technical variables such as size of breast lesions, number of cores and experience of the radiologist performing image-guided biopsies influence the acquisition of appropriate tissue samples of breast lesions and may lead to false negative results. ${ }^{8}$ Currently, no studies have been done in South Africa to assess the diagnostic yield of US-LCNB, which is widely practised in both the private and public sectors.

The objectives of the study were: (1) to determine the critical size of a breast lesion below which US-LCNB proves nondiagnostic; (2) to assess the influence of technical variables on the diagnostic histological yield of breast specimens obtained; such variables include size of lesion, number of cores obtained and level of experience of the radiologist performing the US-LCNB and (3) to evaluate the sensitivity of US-LCNB.

\section{Methods}

Approximately 1800 patients are seen annually at the Addington Hospital breast imaging centre. Interdepartmental meetings between surgical and radiology consultants, registrars and relevant nursing and radiography staff are held weekly to discuss pertinent cases and their selection for US-LCNB. Patients are counselled by the surgical and radiology team, consent obtained by the radiologists for the biopsy, and appointment dates given within 24 hours to 1 week for the procedure. All appointments are recorded manually in a register at the Department of Radiology. Acquired breast biopsy specimens are sent together with the necessary documentation to the histology laboratory. Histology results for those patients who had lumpectomies or mastectomies for malignancy are also captured on the hospital information system.

The charts of all patients who had an US-LCNB over a period of 18 months (March 2011 - September 2012) at Addington Hospital, Durban, KwaZulu-Natal, South Africa, were retrospectively reviewed. Referral for biopsy was based on clinically palpable and occult lesions/microcalcifications with mammogram and ultrasound findings corresponding to Breast Imaging Reporting and Data System (BIRADS) category four or five. The BIRADS classification was not consistently used in the reporting of the imaging findings. None of the palpable lesions had undergone a prior clinicallyguided CNB.

A Toshiba ultrasound machine with a high-frequency linear array transducer of $7 \mathrm{MHz}-14 \mathrm{MHz}$ capability, 14-gauge BARD needle, BARD Magnum biopsy gun and a coaxial technique were used for all the biopsies (Figure 1a and Figure 1b). Figure 2 demonstrates the surgical tray set-up in preparation for the biopsy. The standard protocol at this facility is that a minimum of four cores per lesion should be obtained. No marker placements were done within the lesions. All patients who consented to the procedure, honoured their appointment date and had a formal histological report, were included in the data set.

Data retrieved from the files included patient demographics, technical variables (sonographic size of lesion in millimetres, number of cores, and experience of the radiologist), histology result and immediate post-procedural complications. As the
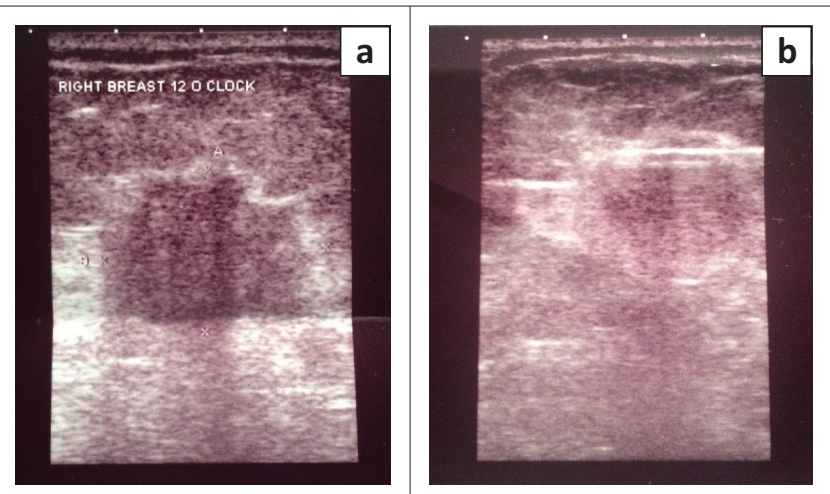

Source: Toshiba ultrasound machine, Department of Radiology, Addington Hospital

FIGURE 1: Sonographic appearance of a histologically proven invasive ductal carcinoma in the right breast of a 51-year-old woman, with (a) irregular margins and shape, hypoechoic with posterior acoustic enhancement (b) a 14-gauge long-throw large core needle with an excursion of $2.2 \mathrm{~cm}$ was used for biopsy under ultrasound guidance.

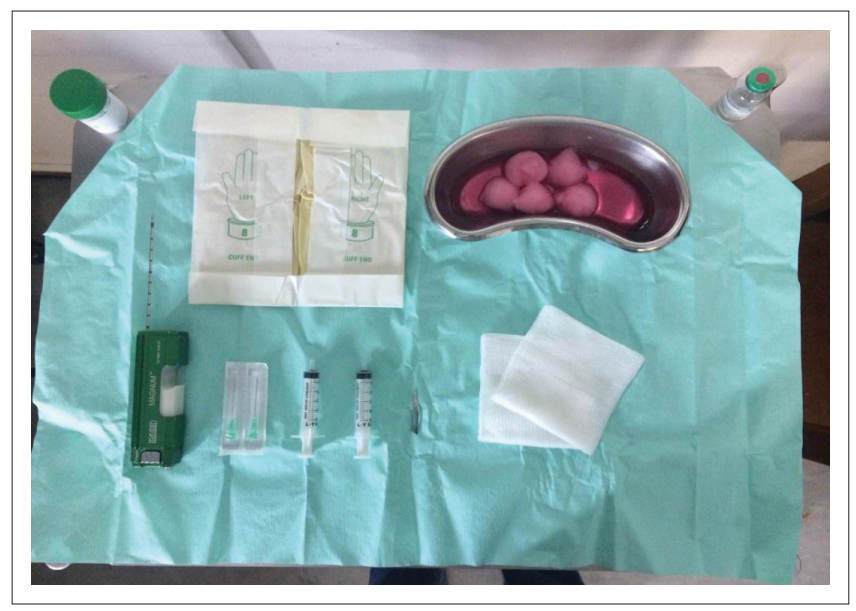

Source: Schene Bhayroo, Department of Radiology, Addington Hospital

FIGURE 2: Sterile biopsy tray set with 14-gauge large core needle attached to a biopsy gun (Bard Medical, Covington, GA, USA) and surgical material. 
focus was on ultrasonographic size of the lesions, no differentiation was made between those that were clinically palpable and those that were occult.

All data were captured in electronic format on an Excel spreadsheet. Descriptive statistics such as means, median and standard deviation were used to summarise data. The independent samples $t$-test and/or the Mann-Whitney test were used to establish if there was a relationship between certain determinant results and technical variables such as size of lesion and the number of cores. Pearson's chi-square and Fisher's exact tests were used to determine association between radiologist's experience and finding determinant results. The level of significance was set at 0.05 .

The study was approved by the Biomedical Research and Ethics Committee of the University of KwaZulu-Natal (BREC Ref: BE187/13). Institutional approval was granted by the Addington Hospital Ethics Committee.

\section{Results}

A total of 147 biopsies were performed over the 18-month period. All patients were female and their mean age was 52 years (range $20-80$ years). Of these, only 132 biopsies were eligible for inclusion in the study owing to missing data for 15 biopsies. All lesions were measured ultrasonographically. There were no documented lesions between $1 \mathrm{~mm}$ and $3 \mathrm{~mm}$, five lesions were between $3.1 \mathrm{~mm}$ and $5.0 \mathrm{~mm}$, and $94 \%$ of the lesions were between $5 \mathrm{~mm}$ and $60 \mathrm{~mm}$ in size, as shown in Table 1.

The overall diagnostic yield was 98.5\% (130/132 biopsies): $71(53.8 \%)$ lesions were malignant including one case of lymphoma; 59 (44.7\%) lesions were benign (one case of Tuberculosis [TB]); and two (1.5\%) lesions were indeterminate, as depicted in Table 2. The latter two patients did not have a definitive histological result as they did not return for follow-

TABLE 1: Lesions biopsied under ultrasound guidance $(N=132)$.

\begin{tabular}{lll}
\hline Lesions & $\boldsymbol{n}$ & $\mathbf{\%}$ \\
\hline $1.0 \mathrm{~mm}-3.0 \mathrm{~mm}$ & 0 & - \\
$3.1 \mathrm{~mm}-5.0 \mathrm{~mm}$ & 5 & 3.8 \\
$5.0 \mathrm{~mm}-60.0 \mathrm{~mm}$ & 124 & 94.0 \\
Microcalcifications & 3 & - \\
Not recorded & 15 & - \\
\hline
\end{tabular}

TABLE 2: Results for ultrasound-guided large core needle biopsy of 132 breast lesions $(N=132)$.

\begin{tabular}{lcc}
\hline Histology results of 132 specimens & $\boldsymbol{n}$ & $\mathbf{\%}$ \\
\hline Malignant & $\mathbf{7 1}$ & $\mathbf{5 3 . 8}$ \\
Lymphoma & 1 & - \\
Carcinoma & 70 & - \\
Benign & $\mathbf{5 9}$ & $\mathbf{4 4 . 7}$ \\
TB of the breast & 1 & - \\
Other & 58 & - \\
Indeterminate & 2 & 1.5 \\
Hamartoma - excision biopsy advised & - & - \\
Papillary neoplasm - excision biopsy advised & - & - \\
\hline
\end{tabular}

TB, Tuberculosis up. Of the 71 malignancies detected non-surgically, 60 were confirmed as malignant at excision. There were no results for 11 excision specimens; these were either from patients who were found to have metastatic disease on staging, or who defaulted their post-biopsy clinic visit and therefore did not have definitive surgery. Therefore the sensitivity for detecting malignancy was $100 \%$.

Figure 3 and Figure 4 demonstrate the malignant and benign lesions, respectively. Invasive ductal carcinoma represented $77 \%$ of all malignancies. No cases of invasive lobular carcinoma were detected. There was one case of lymphoma and one of TB. Of the 132 lesions, 59 were benign at core biopsy. The initial BI-RADS categories for the 59 benign lesions was not reviewed to determine radiologic-pathologic discordance, and continued annual mammographic and sonographic surveillance was recommended.

\section{Biopsy according to size of lesion}

The sizes of 129 lesions were documented by the attending radiologist. In three cases, only microcalcifications were biopsied. The biopsied microcalcifications were: fibroadenoma (benign lesion - junior radiologist - four

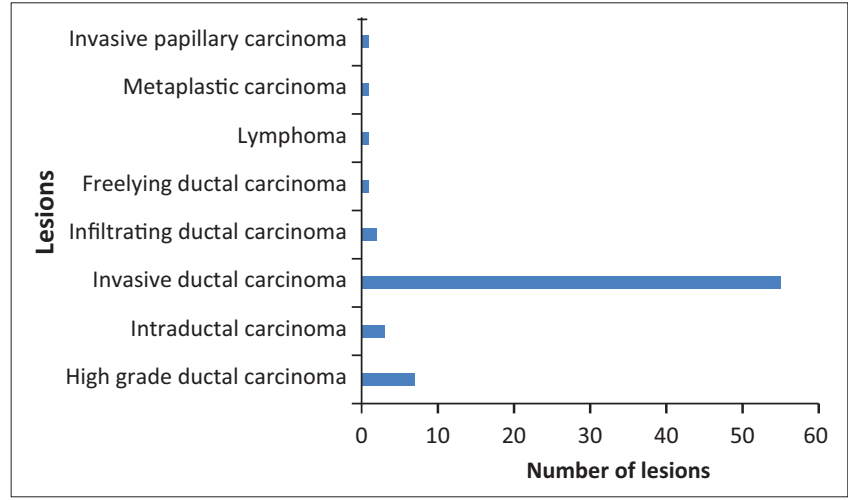

FIGURE 3: Malignant lesions $(N=71)$.

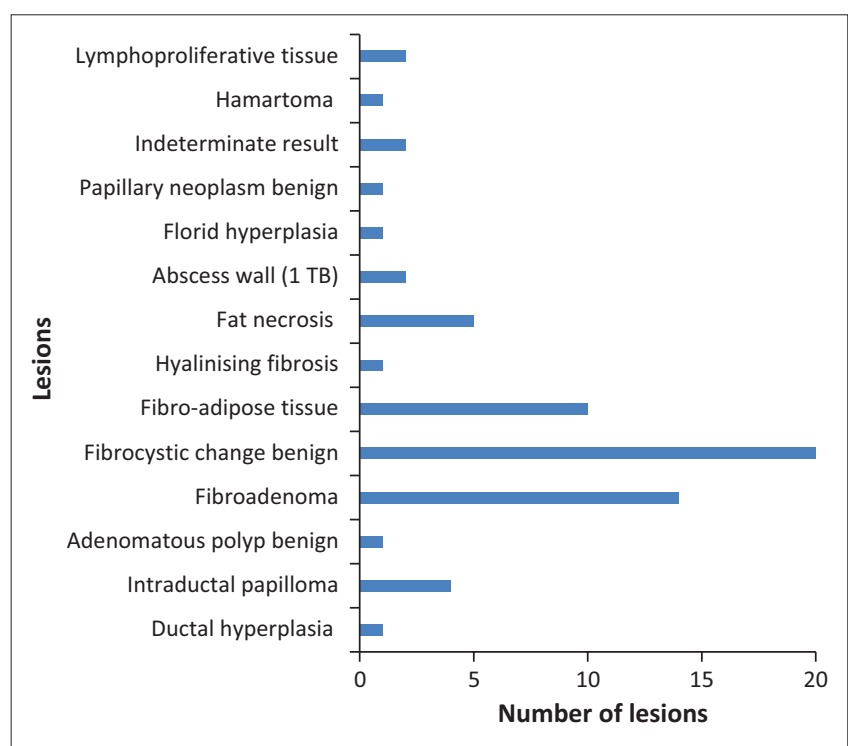

FIGURE 4: Benign lesions $(N=59)$. 
cores); hyalinised fibrous connective tissue (benign lesion senior radiologist - two cores); invasive ductal carcinoma (malignant lesion - senior radiologist - four cores). In the category $3.1 \mathrm{~mm}-5.0 \mathrm{~mm}$, five histology results were as follows: necrotising granulomatous inflammation $(3.1 \mathrm{~mm} /$ benign lesion); invasive ductal carcinoma ( $4 \mathrm{~mm})$; high grade ductal carcinoma in situ $(5 \mathrm{~mm})$; abscess $(5 \mathrm{~mm} /$ benign lesion); and ductal hyperplasia (5 $\mathrm{mm}$ /benign lesion). A minimum of four cores were obtained by the same senior radiologist. There was no statistically significant difference between the various sizes and the eventual biopsy result: all biopsies were diagnostic ( $p=0.065)$.

\section{Biopsy according to number of cores taken}

Of the lesions biopsied, 126 out of 132 (95.5\%) had between four and six cores, five lesions had between one and three cores, and only one lesion had more than six cores, as shown in Figure 5. Of the five lesions with one - three cores, one was indeterminate (60 $\mathrm{mm}$ - one core); one metaplastic carcinoma (20 mm - two cores), one fibroadenoma (19 mm -

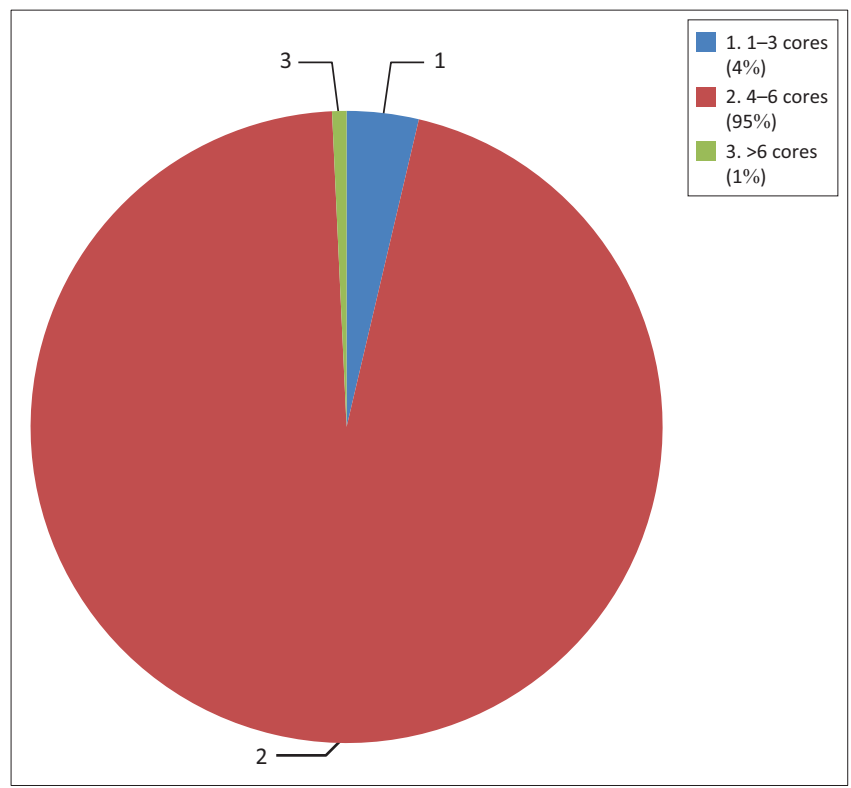

FIGURE 5: Representation of lesions biopsied, tiered according to the number of cores taken.

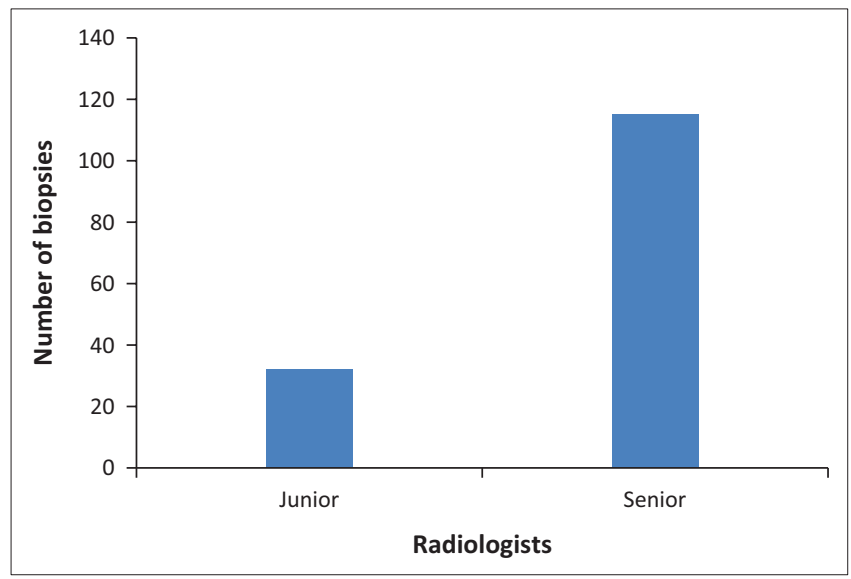

FIGURE 6: Number of biopsies performed by junior and senior radiologists. one core), one fibrocystic change (17 $\mathrm{mm}$ - two cores) and one invasive ductal carcinoma ( $9 \mathrm{~mm}$ - two cores). All were performed by the same senior radiologist and all were $>5 \mathrm{~mm}$ in size. There was no statistically significant difference between the number of cores obtained and the final histology result $(p=0.07)$.

\section{Diagnostic histological yield according to level of experience}

The diagnostic histological yield was $100 \%$ (27 out of 27 biopsies) for the junior radiologists and 98\% (103 out of 105 biopsies) for the senior radiologists (Figure 6), irrespective of the lesion size or the number of cores per lesion $(p=0.085)$. Both indeterminate lesions were biopsied by the same senior radiologist; the larger of the two measured $60 \mathrm{~mm}$ sonographically and had one core biopsy taken, whereas the smaller lesion measured $10.4 \mathrm{~mm}$ and had five cores taken. It is uncertain why only one specimen was taken from the larger lesion although the standard protocol at this breast facility advocates a minimum of four core biopsies per lesion.

\section{Discussion}

The present study demonstrated an overall diagnostic histological yield of $98.5 \%$ and a sensitivity of $100 \%$ for detecting malignancy. This finding is comparable to those of other international reported series. ${ }^{6,7,10,11,12,13}$ Diagnostic tissue biopsy yield is influenced by several factors: number of core specimens obtained, size of core biopsy needle, size of lesion, and experience of the radiologist performing the biopsy.

Wu et al. ${ }^{13}$ demonstrated that at least five specimens should be obtained from suspicious breast lesions in order to achieve an adequate tissue yield; however, other authors recommend at least three biopsy specimens. ${ }^{9}$ In our study, 126/132 (95.5\%) of the lesions biopsied had between four and six cores with only one indeterminate result, which conforms to international standards and contributed to the high diagnostic yield.

Helbich et al. ${ }^{6}$ showed that diagnostic accuracy increased with needles larger than 14 gauge. All the biopsies in this study were performed with a 14 gauge needle.

Wallis et al. ${ }^{14}$ suggested that a competent radiologist should at least have done 20 US-LCNBs under supervision before commencing independent practice, and then a minimum of 25 per year to maintain competence. To the contrary, in the present study, the diagnostic histological yield was 100\% for the junior and $98 \%$ for the senior radiologists. This finding suggests that a prolonged learning curve is probably not necessary to achieve a high diagnostic yield.

There are inconsistent data pertaining to the minimum size of a breast lesion that is amenable to US-LCNB. Schueller et al. ${ }^{8}$ mention that virtually any breast lesion visualised on US can be biopsied using LCNB. Of the 129 measurable 
lesions in our study, 124 (94\%) were $>5 \mathrm{~mm}$ in size, and only five lesions were between $3.1 \mathrm{~mm}$ and $5 \mathrm{~mm}$; amongst these, only one result was indeterminate. The mammogram for this indeterminate result was not reviewed. In our study, there were no lesions $<3 \mathrm{~mm}$. Whilst there is a lack of statistical relevance pertaining to the critical size below which a breast lesion biopsy proves non-diagnostic, four of the five lesions biopsied in the $3.1 \mathrm{~mm}-5.0 \mathrm{~mm}$ category were diagnostic.

\section{Conclusion}

Although our study did not establish a statistically significant relationship between certain technical variables such as size of lesion, number of core specimens obtained, radiologist experience, and histological outcome, the overall diagnostic yield and the sensitivity for detecting malignancy using US-LCNB was comparable to other similar international studies. It is recommended that a prospective study be done; this will determine the limitations of USG-LCNB (if any) as well as the sensitivity and specificity of USG-LCNB in our setting.

\section{Limitations of the study Senior radiologists versus junior radiologists}

Senior radiologists with more than five years' experience / $>25$ biopsies per year performed 105 (79.5\%) of the 132 biopsies. This presented a bias in the statistical analyses with respect to the relationship between variables (i.e. number of cores; size of lesions) and determinant results. The $p$-values were $>0.05$ and proved to be insignificant both individually and combined. Furthermore, the biopsy cores with indeterminate histology results were both taken by the senior radiologists. There were no indeterminate results for the three microcalcificationsbiopsied, and two of themicrocalcifications were biopsied by senior radiologists. Therefore, it is important for radiologists to be aware of technical difficulties that may result in targeting errors. Some of the factors that influence inaccurate tissue sampling include poor lesion or needle visualisation, deep-sited chest wall lesions, mobile lesions, small lesions and haematoma formation obscuring the lesion. ${ }^{15}$

\section{False negative results}

BI-RADS does not provide recommendations on the surveillance of benign lesions at core biopsy, and there are no standardised international guidelines on the frequency and duration of follow-up. Sonographic and mammographic surveillance protocols in the literature range from as early as six months post-biopsy and extend up to two years. . $^{2,8,9,15}$ The present study was limited by the lack of long-term follow-up for all patients with benign biopsies at UG-LCNB. Therefore, false negative rates could not be calculated. Furthermore, the pathologist did not participate in reviewing the benign results. Errors in the initial pathological examination might have been missed.

\section{Radiological-pathological discordance}

As only a BI-RADS score was recorded and the radiological diagnosis of each lesion was not documented by the reporting radiologist, it was not possible to determine radiologicalpathological discordance. A prospective trial should be conducted to address this omission.

\section{Poor record keeping}

Data pertaining to the size of the lesion, number of cores and experience of the radiologist were not recorded for 15 biopsies. These cases could not be included in the study, thus decreasing the sample size.

\section{Acknowledgements Competing interests}

The authors declare that they have no financial or personal relationships which may have inappropriately influenced them in writing this article.

\section{Authors' contributions}

S.B. was responsible for conceptualising the project, drafting the research protocol, completing the data collection and data analysis, and writing the journal article. L.Y.P. made conceptual contributions and assisted with data retrieval and analysis. I.B. made conceptual contributions, supervised and edited the research protocol and final journal article, and facilitated the data collection, analysis and interpretation of results.

\section{References}

1. National Institute for Occupational Health [homepage on the Internet]. Cance statistics, 2009;9-10. [cited n.d.]. Available from http://nioh.ac.za/assets/files/ NCR_2009_FINAL.pdf

2. Parikh J, Tickman R. Image guided tissue sampling: Where radiology meets pathology. Breast J. 2005;11:403-409. http://dx.doi.org/10.1111/j.1075-122x. 2005.00130.x

3. Tabar L, Vitak B, Chen HH, Yen MF, Duffy SW, Smith RA. Beyond randomized controlled trials: Organized mammographic screening substantially reduces breast carcinoma mortality. Cancer. 2001;91:1724-1731. http://dx.doi.org/10. breast carcinoma

4. Kolb TM, Lichy J, Newhouse JH. Comparison of the performance of screening mammography, physical examination, and breast US and evaluation of factors
that influence them: An analysis of 27,825 patient evaluations. Radiology. 2002;225:165-175. http://dx.doi.org/10.1148/radiol.2251011667

5. Leconte I, Feger C, Galant C, et al. Mammography and subsequent whole- breast sonography of nonpalpable breast cancers: The importance of radiologic breast density. AJR Am J Roentgenol. 2003;180:1675-1679. http://dx.doi.org/10.2214/ ajr.180.6.1801675

6. Helbich TH, Rudas M, Haitel A, et al. Evaluation of needle size for breast biopsy: Comparison of 14-, 16-, and 18-gauge biopsy needles. AJR Am J Roentgenol. 1998;171:59-63. http://dx.doi.org/10.2214/ajr.171.1.9648764

7. Pijnappel RM, Van den Donk M, Holland R, et al. Diagnostic accuracy for different strategies of image-guided breast intervention in cases of nonpalpable breast lesions. Br J Cancer. 2004;90:595-600. http://dx.doi.org/10.1038/sj.bjc.6601559

8. Scheuller G, Scheuller WC, Helbich TH. Accuracy of ultrasound-guided, large-core needle breast biopsy. Eur Radiol. 2008;18:1761-1773. http://dx.doi.org/10.1007/ s00330-008-0955-4

9. Liberman L, Feng TL, Dershaw DD, Morris EA, Abramson AF. US guided core breast biopsy: Use and cost effectiveness. Radiology. 1998;208:717-723. http://dx.doi. org/10.1148/radiology.208.3.9722851

10. Buchberger W, Niehoff A, Obrist P, Rettl G, Dunser M. Sonographically guided core needle biopsy of the breast: Technique, accuracy and indications. Radiologe. 2002;42:25-32. http://dx.doi.org/10.1007/s117-002-8113-9

11. Collins LC, Connolly JL, Page DL, et al. Diagnostic agreement in the evaluation of image-guided breast core needle biopsies: Results from a randomized clinical trial. Am J Surg Pathol. 2004;28:126-131. http://dx.doi.org/10.1097/00000478200401000-00015 
12. Memarsadeghi M, Pfarl G, Riedl C, Wagner T, Rudas M, Helbich TH. Value of 14-gauge ultrasoundguided large-core needle biopsy of breast lesions: Own results in comparison with the
doi.org/10.1055/s-2003-37822

13. Wu YC, Chen DR, Kuo SJ. Personal experience of ultrasound-guided 14-gauge core biopsy of breast tumour. Eur J Surg Oncol. 2006;32:715-718. http://dx.doi. org/10.1016/j.ejso.2006.04.012
14. Wallis M, Tardivon A, Helbich T, Schreer I. Guidelines from the European Society of Breast Imaging for diagnostic interventional breast procedures.
Eur Radiol. 2007;17581-17588. http://dx.doi.org/10.1007/s00330-006$0408-x$

15. Youk JH, Kim EK, Kim MJ, Lee YJ, Oh KK. Missed breast cancers at US-guided core needle biopsy: How to reduce them. Radiographics. 2007;27:79-94. http://dx.doi. org/10.1148/rg.271065029 